\title{
Dispositivo con filtro por campo magnético, para retirar polvo metálico en maquina CNC en la industria metalmecánica
}

\section{Device with magnetic field filter, to remove metallic dust in CNC machine in the metalworking industry}

FLORES-GALVÁN, Francisco Ángel †*, NAVA-MORALES, Francisca, ALVA-GALLEGOS, Rodrigo, BALTAZAR-PLATA, Carlos Gustavo

Universidad Tecnológica del Valle de Toluca, Lerma, México.

ID ${ }^{\text {er }}$ Autor: Francisco Ángel, Flores-Galván / ORC ID: 0000-0001-9434-4610

ID $1^{\text {er }}$ Coautor: Francisca, Nava-Morales / ORC ID: 0000-0001-8931-6770, CVU CONACYT ID: 507130

ID $2^{\text {do }}$ Coautor: Rodrigo, Alva-Gallegos / ORC ID: 0000-0002-9354-2006, CVU CONACYT ID: 333455

ID $3^{\text {er }}$ Coautor: Carlos Gustavo, Baltazar-Plata / ORC ID: 0000-0003-4131-7138

\begin{abstract}
Resumen
Objetivo: Implementar el "filtro por campo magnético" y poder mejorar la calidad de las piezas geométrica y visual, devolviendo las propiedades al refrigerante (aceite) quitándole las impurezas, y así poder mejorar la eficiencia de los maquinados mediante este proyecto. Metodología: El dispositivo está integrado por un filtro por campo magnético que fue diseñado para retirar las micro partículas metálicas que se encuentran en el fluido refrigerante y con esto disminuir la variación geométrica y dimensional en las especificaciones de piezas de precisión maquinadas en CNC. Se Aplica un sistema de filtración de la rebaba del aceite refrigerante por medio de un electroimán. La finalidad de este proyecto es bajar la cantidad de Scrap por variación producida en las medidas finales por el desgaste prematuro del filo de la herramienta de corte generado por estas partículas metálicas suspendidas en el aceite refrigerante aun después de ser filtrado convencionalmente. Con este proyecto se pretende también alargar el tiempo de vida útil de las herramientas (insertos, brocas y machuelos), ya que la durabilidad de las mismas se ve disminuida y al perder el filo se fracturan prematuramente produciendo piezas scrap. Contribución: La implementación del filtro permitiera reducir en un 90 por ciento el defecto de piezas marcadas por residuos dentro de refrigerante.
\end{abstract}

Filtro, Campo magnético, Maquina CNC

\begin{abstract}
Objective: Implement the "filter by magnetic field" and be able to improve the quality of the geometric and visual pieces, returning the properties to the refrigerant (oil) by removing the impurities, and thus being able to improve the efficiency of the machining through this project. Methodology: The device is integrated by a magnetic field filter that was designed to remove the metallic microparticles found in the refrigerant fluid and thereby reduce the geometric and dimensional variation in the specifications of precision machined parts in CNC. A filtering system for the burr of the refrigerant oil is applied by means of an electromagnet. The purpose of this project is to reduce the amount of Scrap by variation produced in the final measurements by premature wear of the cutting tool edge generated by these metal particles suspended in the cooling oil even after being filtered conventionally. This project also aims to extend the useful life of the tools (inserts, bits and shanks), since the durability of the tools is reduced and when they lose the edge they fracture prematurely producing scrap pieces. Contribution: The implementation of the filter would reduce the defect of parts marked by residues inside the refrigerant by 90 percent.
\end{abstract}

Filter, Magnetic field, CNC Machine

Citación: FLORES-GALVÁN, Francisco Ángel, NAVA-MORALES, Francisca, ALVA-GALLEGOS, Rodrigo, BALTAZAR-PLATA, Carlos Gustavo. Dispositivo con filtro por campo magnético, para retirar polvo metálico en maquina CNC en la industria metalmecánica. Revista del Diseño Innovativo. 2019. 3-7: 20-25

\footnotetext{
* Correspondencia del Autor (Correo electrónico: francisco.flores@utvtol.edu.mx)

$\dagger$ Investigador contribuyendo como primer autor.
} 


\section{Introducción}

Los procesos de manufactura son la combinación de personal, materia prima, herramientas y máquinas para la obtención de un producto final que cumple con las especificaciones requeridas. Uno de los procesos de manufactura más usados en la producción de piezas metálicas es el corte con desprendimiento de material (rebaba), conocido como maquinado o mecanizado. Es decir, una herramienta con filos cortantes fabricada de un material resistente al desgaste y a las altas temperaturas, penetra la superficie de una pieza con desprendimiento de material en forma de viruta, hasta obtener el producto final. Los parámetros fundamentales en un proceso de corte son: velocidad de corte, avance y profundidad.

La velocidad de corte (Vc) es el camino recorrido por el filo de la herramienta por unidad de tiempo. Donde $\mathrm{D}$ es el diámetro de la pieza y $\mathrm{n}$ es la velocidad de rotación. $\mathrm{Vc}=\pi^{*} \mathrm{D}^{*} \mathrm{n}(1)$ Actualmente las máquinas herramientas usadas en los procesos de mecanizado vienen altamente automatizadas, reduciendo las necesidades de mano de obra para ciertos procesos productivos. Estas máquinas se pueden programar usando el control numérico computarizado (CNC), dichos programas contienen la información tecnológica sobre el proceso de mecanizado.

También se pueden programar usando la ayuda del diseño asistido por computador (CAD), donde se diseñan las partes a mecanizar. Posteriormente pasan a un programa de manufactura asistida por computador (CAM) donde se realiza la simulación del proceso de corte, seleccionando las herramientas, materiales y parámetros de corte. Los programas CAD/CAM ayudan a realizar programas $\mathrm{CNC}$ complejos.

La industria metalmecánica está adquiriendo máquinas herramientas de última tecnología, pero muchas de éstas no son aprovechadas al máximo por las empresas, debido a que las velocidades no se optimizan y los materiales de las herramientas de corte no siempre son los más adecuados y la rebaba que se adhiere en la herramienta provoca su degradación prematura.

Las herramientas de corte deben cumplir con tres propiedades importantes: tenacidad, dureza en caliente y resistencia al desgaste.
Un material para herramienta ampliamente usado desde principios del siglo XX es el acero de alta velocidad o acero rápido (HSS), se ha seguido usando debido a que se han hecho mejoras en los ingredientes de aleación y por su bajo costo. Las herramientas de acero de alta velocidad son reafilables, ya que todo el vástago es del mismo material.

Actualmente existen nuevos materiales para herramientas con mejores propiedades mecánicas, como son los carburos cementados y los cerámicos. Estos materiales son desechables y se fijan mecánicamente en un portaherramientas. La vida de la herramienta es un factor importante para la reducción de los costos de mecanizado y se puede calcular con base en la ecuación de Taylor:

$\mathrm{Vc} * \mathrm{Tn}=\mathrm{C}$

Donde $\mathrm{T}$ es la vida de la herramienta, $\mathrm{n}$ y C son parámetros que dependen del avance, la profundidad de corte y especialmente del material dela herramienta.

La vida de la herramienta por la adherencia del polvo metálico se ve disminuida aumentando los costos de mecanizado.

En la empresa donde se implementara este prototipo elabora piezas con un grado de dificultad variado en cada una de sus medidas y se tiene variación en las especificaciones principalmente por los factores como son:

\footnotetext{
- Materia prima.

- Tipo de Herramientas de corte (insertos, brocas).

- Rebaba.

- Aceite contaminado con pequeñas partículas de rebaba (polvo metalico).
}

Lo que ocasiona variación en sus medidas pudiendo provocar piezas de scrap en el área. El proceso y procedimiento es fundamental si no se llevan a cabo correctamente, no podemos cumplir las políticas de calidad de la empresa, afectando lel estándar de producción y no satisfaciendo las necesidades del cliente.

Se implementara el uso del "filtro por campo magnético" con la finalidad de retirar el polvo metálico del refrigerante utilizado $\mathrm{y}$ aprovechar la eficiencia de la máquina. 


\section{Problema}

La empresa en la que se pretende implementar este proyecto se dedica a la fabricación de piezas maquinadas de alta precisión, para la industria automotriz, aeronáutica, aeroespacial entre otras. Principalmente las piezas automotrices son las que aportan y dan la mayor utilidad a la empresa.

En estos procesos se ha suscitado el problema de piezas marcadas en su geometría por fragmentos ajenos a las mismas, cabe mencionar que gracias a esto se tienen varios inconvenientes en cuanto a la exactitud de medición en la geometría de las piezas, y del maquinado entre otros factores que impiden que estas piezas sean aceptadas como piezas buenas. Y como principal factor a combatir se encontró que el refrigerante es por donde se filtran muchas de estas partículas.

Es por eso que se recurrió a la elaboración de un electroimán el cual nos ayudara a detener la mayor cantidad de rebaba que pueda encontrarse dentro de nuestro lubricante para de esta manera evitar que las piezas tengan cierto nivel de variación.

Se espera que el proyecto logre reducir más de un $80 \%$ o en su totalidad las marcas dentro del proceso de fabricación de los diferentes números de parte.

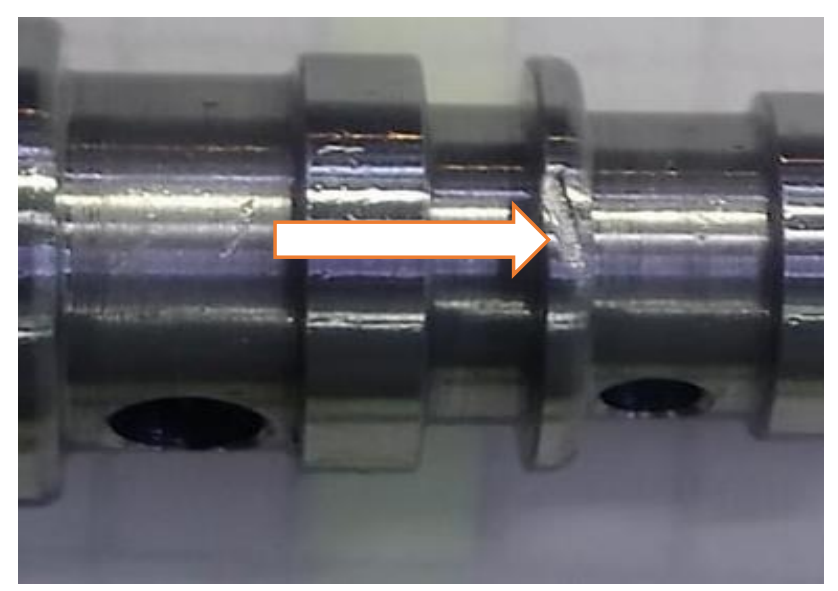

Figura 1 Ejemplo de una pieza con marca

\section{Objetivo General}

Implementar el "filtro por campo magnético" y poder mejorar la calidad de las piezas geométrica y visual, devolviendo las propiedades al refrigerante (aceite) quitándole las impurezas, y así poder mejorar la eficiencia de los maquinados mediante este proyecto.

\section{Desarrollo}

El diseño del proyecto implementado en la empresa para lograr reusar el aceite refrigerante contaminado en el proceso.

La estructura llevara dos filtros por donde pasara el aceite.

El primer paso del sistema de filtración es una coladera donde se filtrara la rebaba más grande que se llegue a pasar.

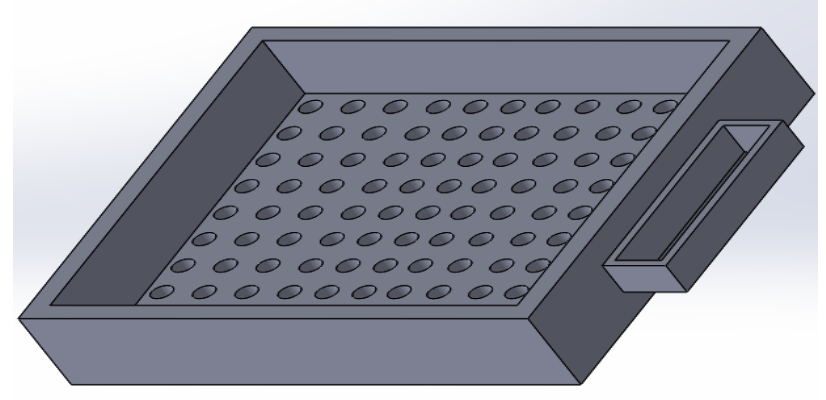

Figura 2 Charola superior

El segundo paso del sistema de filtración será un recipiente que va a estar eletroimantado por medio de un transformador modificado para obtener el proceso de electroimán.

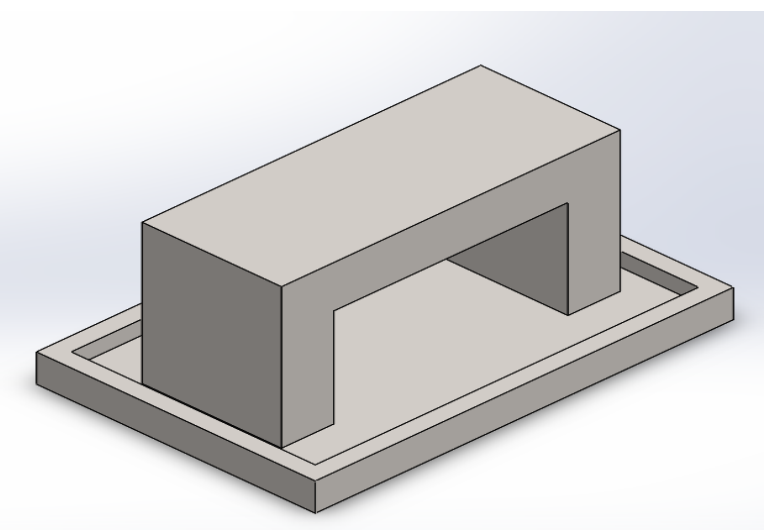

Figura 3 Bandeja retirable imantada

La estructura del proyecto esta conformada por una canaleta para hacer mas rapido el proceso de filtracion.

La forma delgada de la estructura es lo que hace más factible la limpieza de los filtros no afecta las operaciones de maquinado y no interfiere en las actividades manuales del operador. En la parte superior de la canaleta se colocara la charola para captar la rebaba de mayor tamaño La estuctura de la canaleta permite que el aceite caiga por gravedad.

FLORES-GALVÁN, Francisco Ángel, NAVA-MORALES, Francisca, ALVA-GALLEGOS, Rodrigo, BALTAZAR-PLATA, Carlos Gustavo. Dispositivo con filtro por campo magnético, para retirar polvo metálico en maquina CNC en la industria metalmecánica. Revista del Diseño Innovativo. 2019 


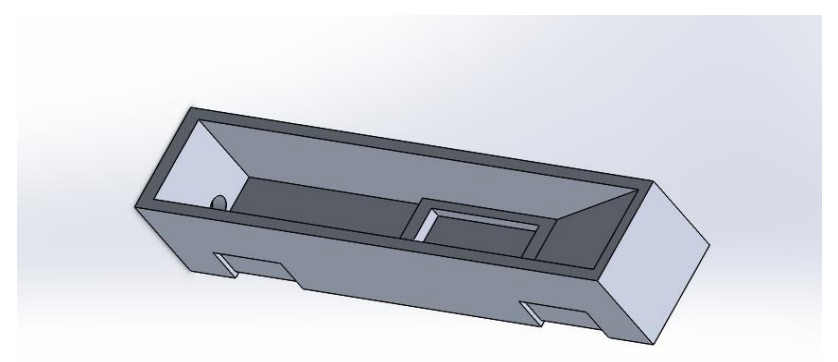

Figura 4 Canaleta inferior

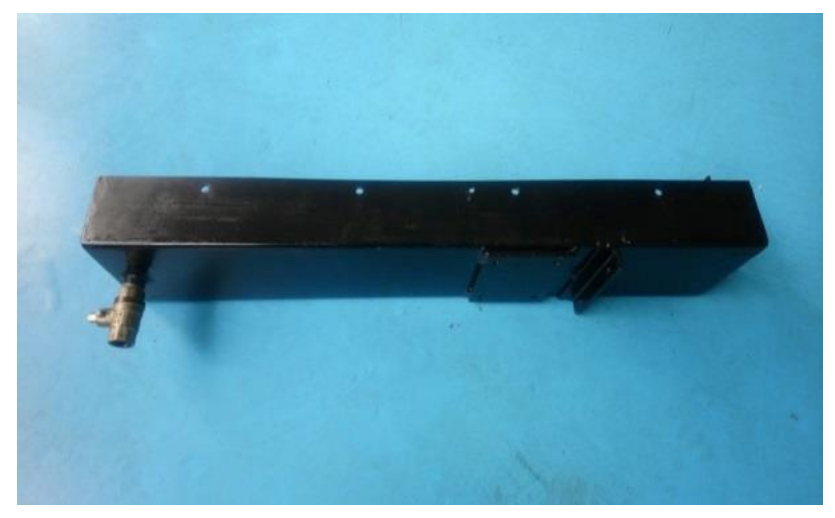

Figura 5 Canaleta inferior

El transformador deberá de ir en la parte inferior de la canaleta y de esta manera la canaleta pueda ser imantada y las partículas puedan ser atrapadas.

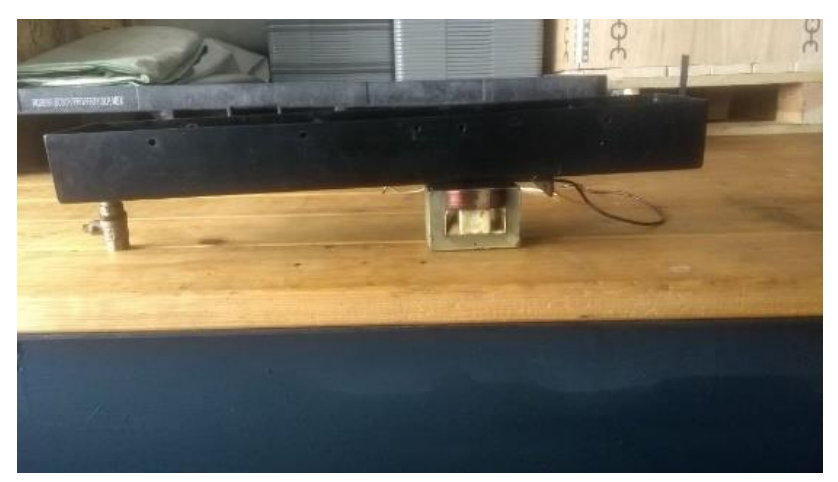

Figura 6 Transformador ensamblado en la canaleta inferior

La estructura cuenta con un conducto superior que será por donde el refrigerante se introducirá inicialmente $\mathrm{y}$ en donde las partículas de gran tamaño serán detenidas para después pasar a la canaleta inferior. Este conducto tiene una ranura donde se colocara la charola perforada como se muestra en la fig. 7.

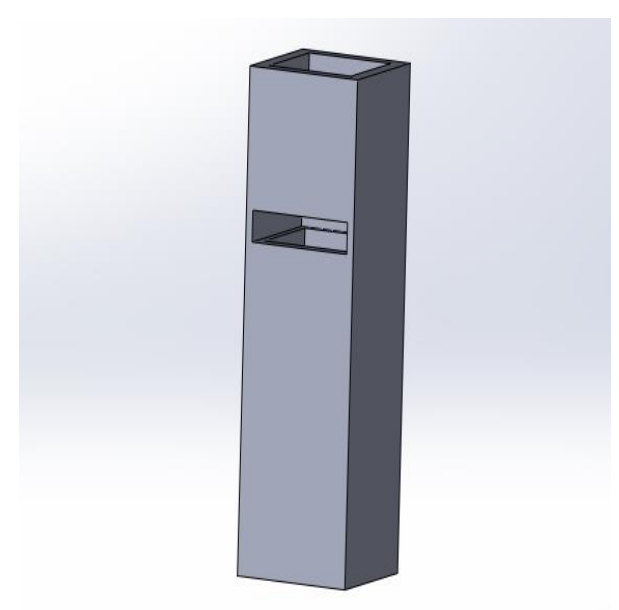

Figura 7 Conducto superior

El sistema de control en nuestro proyecto contendrá un botón selector de encendido que controlara el transformador y a la vez accionara la bamba del aceite, otro botón de reset, y un paro de emergencia. Así como también 3 focos que nos indicaran el estado del equipo. Un rojo de paro, un verde de encendido y un amarillo de falla. Como se muestra en la imagen.

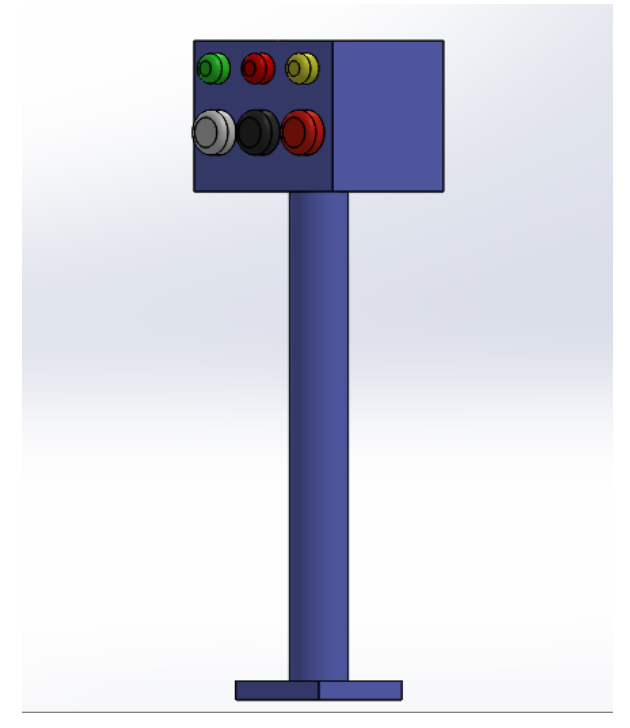

Figura 8 Sistema de control botonera

La altura total del proyecto será de 1.80 metros de altura como se muestra fig.9. 


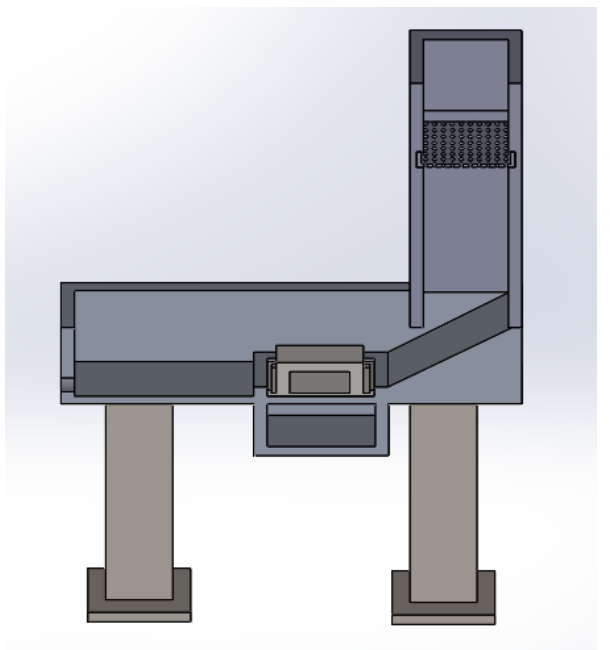

Figura 9 Ensamble final

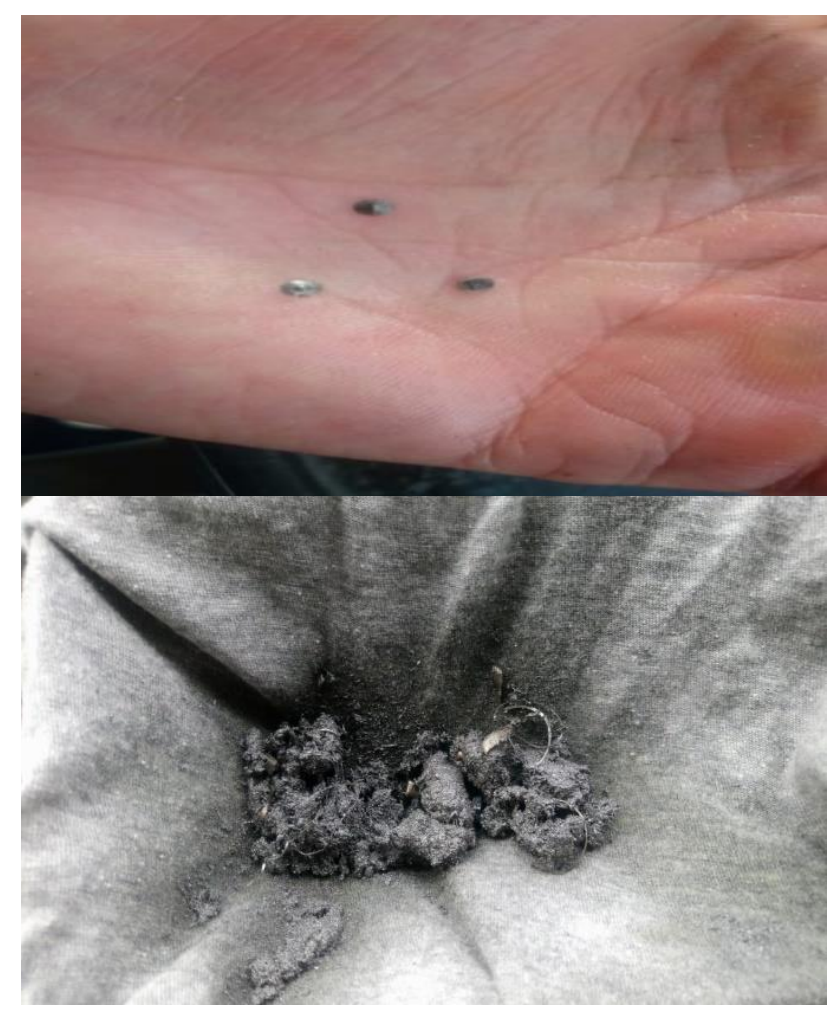

Figura 10 Ejemplo de algunas partículas que han sido atrapadas por el magnetismo

En las primeras pruebas que se realizaron se logró captar una gran cantidad de polvo metálico.

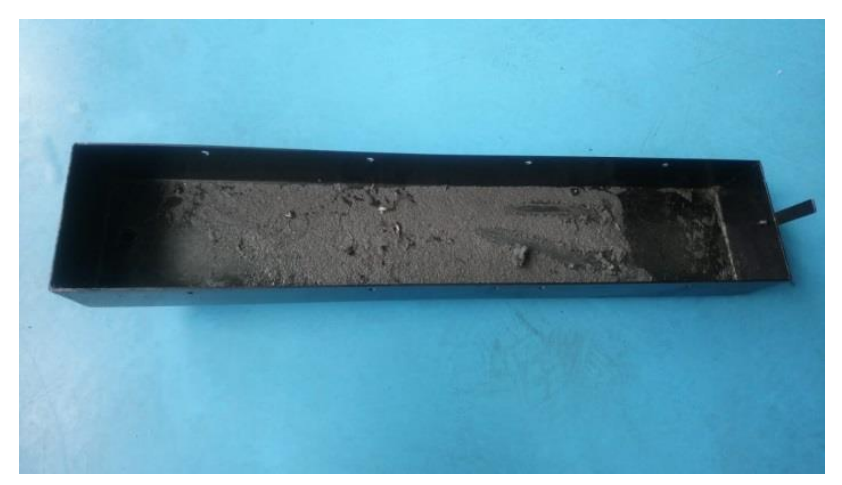

Figura 11 Ejemplo de los residuos atrapados en la canaleta
Por políticas de privacidad y confidencialidad de la empresa donde se realizó el dispositivo no fue posible mostrar todos los componentes involucrados solo las partes más relevantes y autorizadas.

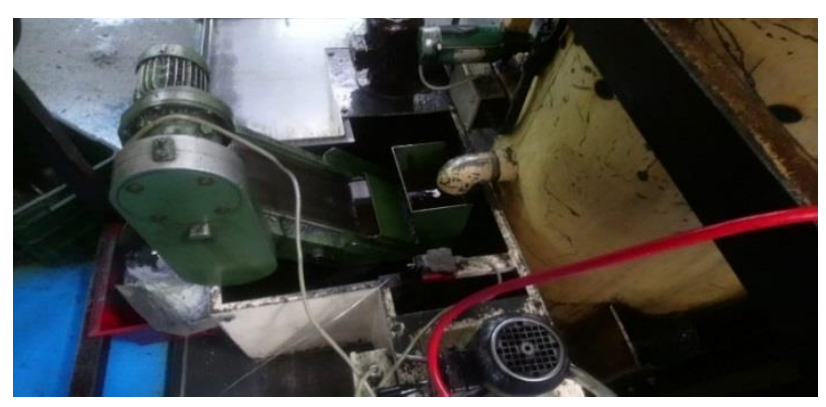

Figura 12 Lugar donde se implementara el proyecto finalmente

\section{Resultados}

La implementación del electroimán como filtro mejoro la calidad de las piezas ya que los filos de la herramienta son más durables ya que las partículas de rebaba actúan como abrasivos reduciendo su vida útil y en consecuencia no se logra la geometría y el acabado especificado en las piezas maquinadas además al contaminarse menos el refrigerante conserva sus propiedades por más tiempo y su principal objetivo que es: la reducción de piezas que estén fuera de especificación.

La implementación de este proyecto ayuda a reducir en un $90 \%$ por ciento el defecto de piezas marcadas por residuos dentro de refrigerante. Además una mejora que no se tenía contemplada pero que se suscitó gracias a la implementación del proyecto fue duplicar la vida del filtro de alta presión.

\section{Análisis de costo - beneficio / tiempo de retorno}

Existen diversas maquinas o equipos que nos garantizan el centrifugado de la viruta generada por tornos, rectificadoras $\mathrm{y}$ otras máquinas. Garantizándonos un mejor secado de rebaba para su venta después del maquinado. El problema de estas centrifugadoras no, nos garantizan un refrigerante limpio de rebaba, ya que aún se encuentran pequeñas partículas de rebaba en el refrigerante y este es reutilizado en el proceso, en estas condiciones nos ocasiona algunos problemas en el maquinado de las piezas.

FLORES-GALVÁN, Francisco Ángel, NAVA-MORALES, Francisca, ALVA-GALLEGOS, Rodrigo, BALTAZAR-PLATA, Carlos Gustavo. Dispositivo con filtro por campo magnético, para retirar polvo metálico en maquina CNC en la industria metalmecánica. Revista del Diseño Innovativo. 2019 
El sistema de filtración será capaz de separar una mayor cantidad de las partículas de rebaba del aceite gracias a los dos filtros implementados. Otra ventaja del sistema de filtración, es que no necesita filtros de tela o de algún tipo similar. Para el cálculo del costo se dividió en cuatro aspectos importantes

\begin{tabular}{|l|r|}
\hline \multicolumn{1}{|c|}{ Concepto } & \multicolumn{1}{c|}{ Costo } \\
\hline Costo de materiales & $1,710.00$ \\
\hline Costo de mano de obra directa & $1,867.18$ \\
\hline Costo de herramienta & $1,502.00$ \\
\hline Costos de insumos de fabricación & 292.00 \\
\hline Costo Total & $\$ 5,371.18$ \\
\hline
\end{tabular}

Tabla 1 Representación costo de componentes Fuente: Elaboración Propia

Existen diversas filtradoras de aceite en el mercado que ofrecen una buena calidad en los refrigerantes, quitando los residuos de este que se generan por medio del entorno donde se encuentra pero el precio de ellas oscila de 20,000 a 100,000 pesos. Pero se requiere tener un área específica para su instalación lo que generaría hacer un estudio de distribución de planta lo más adecuado posible. Con ayuda de los datos recabados podemos establecer el estudio costo beneficio que tendrá el proyecto, lo cual nos dirá si es aceptable o no y ver si puede ser implementado en más maquinaria.

$\frac{\text { Benefico }}{\text { costo }}=\frac{\mathbf{5 0 , 0 0 0}}{\mathbf{6 , 0 0 0}}=8.333$

El proyecto es rentable.

\section{Conclusiones}

El proyecto va a mejorar el proceso de maquinado de las piezas disminuyendo el Scrap, elimina casi en su totalidad las marcas de rebaba, alargando la vida de las herramientas de corte utilizadas en los procesos de fabricación de las diferentes piezas.

La implementación del proyecto ofrece:

- Dos sistemas de filtración que garantizan la limpieza del refrigerante.

$\mathrm{Su}$ diseño es compacto.

- $\quad$ Fácil manejo por su simplicidad ya que su sistema de control estará controlado con un botón selector.

- De igual forma elimina los gastos relacionados con filtros desechables tales como (filtros de tela, papel porosos, etc.) ahorrando en estos insumos.

\section{Referencias}

[1] Roldan Viloria, José (2012) Tecnología y circuitos de aplicación de neumática, hidráulica y electricidad. Thomson, Paraninfo ( $\left({ }^{\mathrm{a}}\right.$ Edición).

[2] Barco Ríos, Héctor, Rojas Calderón, Edilberto, Restrepo Parra, Elisabeth (2012) Principios de Electricidad y Magnetismo. Universidad Nacional de, Colombia. Primera edición

[3] Grupo Pandora S.A (2001) Campos Eléctricos y Magnéticos de $50 \mathrm{~Hz}$. UNESA. Primera edición. 